\title{
High-Pressure High-Temperature Structural Properties of Urea
}

\author{
Kamil Dziubek $^{a, b}$, Margherita Citroni ${ }^{a, c}$, Samuele Fanetti ${ }^{a, d}$,
} Andrew B. Cairns ${ }^{e}$, Roberto Bini ${ }^{a, c, d, *}$

${ }^{a}$ LENS, European Laboratory for Non-linear Spectroscopy, Via N. Carrara 1, I-50019 Sesto Fiorentino, Firenze, Italy

${ }^{b}$ Faculty of Chemistry, Adam Mickiewicz University, Umultowska 89b, 61-614 Poznań, Poland

c Dipartimento di Chimica "Ugo Schiff" dell'Università degli Studi di Firenze, Via della Lastruccia 3, I-50019 Sesto Fiorentino, Firenze, Italy

${ }^{d}$ Istituto di Chimica dei Composti OrganoMetallici, CNR-ICCOM, 50019 Sesto Fiorentino, Italy

e European Synchrotron Radiation Facility, 71 Avenue des Martyrs, 38043 Grenoble, France 


\begin{abstract}
Angle dispersive X-ray diffraction (ADXRD) and Fourier transform infrared (FTIR) spectroscopy have been employed to study the phase diagram of urea crystal beyond $15 \mathrm{GPa}$ and at temperatures in excess of $400 \mathrm{~K}$. Previously reported Bridgman phase II was structurally characterized for the first time, and it is discovered that it coincides with room temperature phase IV. Large metastability P$\mathrm{T}$ regions were identified for all phases in the sequence I-III-IV-V, ascribed to the difficulty to disrupt the H-bonding network, a prerequisite to accomplish the molecular rearrangement necessary for the structural transformation. High-temperature studies and use of a hydrostatic compression medium allows the thermodynamic boundaries of phase III, and partly of phase IV, to be identified so making a considerable step forward in the knowledge of the phase diagram of urea.
\end{abstract}




\section{Introduction}

Urea - also known as carbamide, the amide of carbamic acid - is at ambient conditions a colorless solid highly soluble in water. Urea is one of the most studied molecules because of its biological and chemical importance. It is the final metabolic product of aminic nitrogen in ureotelic organisms, and in fact was the first organic molecule synthesized from "inorganic" compounds (ammonia and cyanic acid); industrially it is an important fertilizer and the starting chemical in the production of drugs and plastics. From a chemicophysical point of view, urea is an archetypal hydrogen-bonded molecular crystal. ${ }^{1}$ Rich polymorphism is exhibited by this crystal with increasing pressure ${ }^{2}$ evidencing the strong interplay between solid-state phase transitions and hydrogen-bond topology. The hydrogen-bonding strength and geometry can indeed be significantly modified by the application of an external pressure and, besides structural changes, this could have important effects on physico-chemical properties, ${ }^{3,4}$ as well as on the chemical reactivity. ${ }^{5,6}$

At ambient pressure the crystal structure of urea is tetragonal, space group $P \overline{4} 2_{1} m\left(D_{2 \mathrm{~d}}^{3} ; Z=2\right)$ (phase $\left.\mathrm{I}\right)$, with the carbonyl group of each molecule forming four $\mathrm{H}$-bonds with three different neighbouring molecules realizing square tunnels with a side of $3.94 \AA$ directed approximately along the $c$ axis. ${ }^{7}$ Compressing phase I at ambient temperature a transition to phase III (Bridgman notation, ref. 8) is observed at $0.48 \mathrm{GPa}$, and both single crystal X-ray diffraction ${ }^{2}$ and neutron powder diffraction ${ }^{9,10}$ agree on an orthorhombic structure, space group $P 2_{1} 2_{1} 2_{1}\left(D_{2}^{4} ; Z=4\right)$. Consistency of the X-ray diffraction data with the space group $P n a 2_{1}\left(C_{2 \mathrm{v}}^{9} ; Z=4\right)$ was also proposed in a combined Raman and X-ray scattering study. ${ }^{11}$ With further 
compression two additional phase transitions were observed. The first, at 2.8 GPa to phase IV (orthorhombic $P 2_{1} 2_{1} 2 ; D_{2}^{3} ; Z=2$ ), was reported both in single crystal X-ray diffraction ${ }^{2}$ and neutron powder diffraction ${ }^{10,12,13}$ experiments. The second, at $7.2 \mathrm{GPa}$ to phase V (orthorhombic Pmcn; $D_{2 \mathrm{~h}}^{16}$; $Z=4$ ), has been so far reported in neutron diffraction ${ }^{12,13}$ and $\operatorname{Raman}^{11}$ experiments. In the Raman study the transition pressures were slightly higher in pressure, 5.0 and $8.0 \mathrm{GPa}$ for the III-IV and IV-V transitions, respectively, and crystal domains with significantly different spectral features were detected in the two phases. ${ }^{11}$

As extensively discussed by Olejniczak et al.,$^{2}$ the molecular arrangement in all these phases is driven by $\mathrm{NH}$. . O hydrogen bonds. The $\mathrm{H}$ bond coordination of phase I reduces in phase III where the carbonyl group interacts with two neighbouring molecules through three $\mathrm{O} \cdots \mathrm{H}$ bonds. This is ascribed to a collapse of the channel voids which also characterizes the higher pressure phase IV. On going from phase III to IV the molecules rearrange so recovering the fourfold coordination of the O-atom, the twofold axis crystal site symmetry, and the alignment along [001] proper of phase I. All these characteristics make phase IV the collapsed version of phase I. ${ }^{2}$

In contrast to the several high-pressure studies at room temperature, there are no structural or spectroscopic studies at high-pressure and hightemperature but only compressibility measurements performed by Bridgman exactly one century ago. ${ }^{8}$ In this study Bridgman reports that urea transforms to a new phase (II) above $373 \mathrm{~K}$ and $0.60 \mathrm{GPa}$, but that the transition line between phases II and III could not be determined because of the small volume change. The high-temperature high-pressure behaviour of urea is 
also an unexplored field from computational point of view. The ability to reproduce experimental crystal phases of urea has been found to be rather limited. Density functional theory (DFT) calculations were used to monitor structural changes of the urea molecule up to $10 \mathrm{GPa}$ under the assumption that no phase transitions occur in this pressure range, therefore maintaining the molecular arrangement characterizing phase I. ${ }^{14}$ Another study based on a crystal potential accounting for van der Waals and long-range Coulomb interactions among the molecules did provide a sequence of phase transitions involving the correct structures of phases III and IV, but in the reverse order. ${ }^{15}$ Finally, new crystal structures were also predicted for pressures in excess of $500 \mathrm{GPa}$ resulting from the pressure induced chemical transformation of ammonium cyanate. ${ }^{16}$

In this study we have performed a combined X-ray diffraction and FTIR absorption spectroscopic study aimed to improve our knowledge of the highpressure and high-temperature behaviour of urea. In particular, we have investigated the structure and the stability range of the high-temperature high-pressure phase II, revealing that this phase coincides with phase IV and that the pressure induced transformation at ambient temperature is kinetically controlled. A new phase diagram and the ambient temperature equation of state (EOS) for phase IV have been obtained.

\section{Experimental}

Crystalline urea was obtained from Aldrich in high purity $(\geq 99 \%)$ and was prepared for high-pressure experiments in the following ways. For FTIR 
experiments, the as-received sample was finely ground and loaded in a membrane diamond anvil cell (MDAC), equipped with IIa type diamonds and rhenium gaskets, without any compression medium. For synchrotron X-ray diffraction experiments neon was used as the pressure transmitting medium (PTM) within the MDAC in all cases. Small single crystals (large face about $20 \times 20 \mu \mathrm{m}^{2}$ and thickness $\left.<10 \mu \mathrm{m}\right)$ were selected for single crystal X-ray diffraction experiments. A couple of small ruby chips were also loaded with the sample for pressure calibration by the ruby fluorescence method. ${ }^{17}$ Hightemperature experiments were performed using resistively heated MDAC cells. In these experiments temperature was measured with a precision of $\pm 0.1 \mathrm{~K}$ using a K-type thermocouple placed close to the diamonds.

Infrared absorption measurements were performed with a Bruker-IFS 120 HR spectrometer suitably modified for high pressure experiments ${ }^{18,19}$ with an instrumental resolution better than $1 \mathrm{~cm}^{-1}$. ADXRD experiments were performed at the ESRF high-pressure beamline ID27 using monochromatic synchrotron radiation of wavelength $\lambda=0.3738 \AA$. Data were collected as a function of temperature and pressure using a MARCCD 165 detector positioned approximately $223 \mathrm{~mm}$ from the sample, as calibrated with a $\mathrm{CeO}_{2}$ standard. The focal spot (FWHM) of the beam was $\sim 3 \mu \mathrm{m}$. For high temperature ADXRD experiments a vacuum chamber equipped with Mylar windows was used. The $2 \mathrm{D}$ diffraction patterns were integrated using DIOPTAS; ${ }^{20}$ manual background subtraction (including the contribution from the diffraction of Mylar windows) was done in Fityk. ${ }^{21}$ Rietveld refinements and LeBail fits were done using the program JANA2006. ${ }^{22}$ While Rietveld refinements accounted for observed ADXRD patterns within an acceptable accuracy in 
most cases, robust refinement was not possible for all data due to (i) the weakly scattering sample, (ii) diamond Compton and (iii) Mylar scattering contributions to the integrated diffraction pattern. We have therefore opted to use LeBail analysis for several data sets.

\section{Results}

\subsection{Diffraction data}

Synchrotron X-ray diffraction experiments were performed both on single crystals and powdered samples with Ne used as the pressure transmitting medium. Powdered samples were compressed at ambient temperature up to $4.1 \mathrm{GPa}$, observing a modification of the diffraction patterns fully consistent with the transitions sequence I-III, III-IV and the crystal structures reported in ref. 2. However, despite the hydrostaticity of neon up to 15-16 GPa (refs. 17,23), we observed in both cases large pressure intervals with coexistence of phases, especially for the III-IV phase transition. The coexistence of the diffraction peaks of phase III well within the pressure domain of phase $\mathrm{IV}$, according to the transition pressure reported in the literature, ${ }^{2,10,12,13}$ is shown in trace (a) of Figure 1. This coexistence is further emphasized by the behaviour observed on heating the sample. In fact, on increasing the temperature at $4.1 \mathrm{GPa}$, the diffraction pattern is still characterized by the presence of the peaks of both phase III and IV up to $422 \mathrm{~K}$ (see trace (b) of Figure 1). This temperature is much higher, by about $50 \mathrm{~K}$, than the temperature required to obtain Bridgman phase II close to the I-II-III triple point. ${ }^{8}$ Therefore, we released the pressure at this temperature to probe the transi- 
tion to Bridgman phase II. The pattern suddenly and appreciably changed around $2.3 \mathrm{GPa}$ (see trace (c) of Figure 1) and the peaks could be successfully and completely assigned using the orthorhombic $P 2_{1} 2_{1} 2$ crystal structure of phase IV. The temperature was slowly lowered, not fully compensating for the resulting small pressure drop, which lead the sample at ambient temperature at $1.4 \mathrm{GPa}$. No changes were observed in the diffraction pattern during this recovery to ambient temperature (see trace (d) of Figure 1), thus indicating that Bridgman phase II is the thermodynamic stable phase at ambient temperature. Bridgman phase II was then compressed at ambient temperature but no changes in the diffraction pattern were observed around $3 \mathrm{GPa}$ where the III-IV transition is reported. ${ }^{2,10,12,13}$ On the contrary, a clear change was observed to start around $6 \mathrm{GPa}$ and complete around $8 \mathrm{GPa}$ where a pattern consistent with that expected for phase $\mathrm{V}$ was obtained. The powder quality and the employment of a hydrostatic compression medium rule out any possible metastability, implying that Bridgman phase II coincides with phase IV. This conclusion was also advanced by Olejniczak et $a l .{ }^{2}$ who speculated that phase IV and phase II are the same phase on the basis of the close relationship between phases I and IV.

The lattice parameters and the volume evolution with pressure of phase IV are reported in Figure 2 with the volume data fitted by a Vinet equation of state: ${ }^{25}$

$$
P=3 B_{0} \frac{\left(1-f_{\nu}\right)}{f_{\nu}^{2}} \exp \left[\frac{3}{2}\left(C_{0}-1\right)\left(1-f_{\nu}\right)\right],
$$

where $f_{\nu}=\left(V / V_{0}\right)^{1 / 3}$, being $V_{0}$ the cell volume at ambient pressure, $B_{0}$ and $C_{0}$ are the isothermal bulk modulus and its derivative against pressure at 
$p=0$, respectively. Contrary to what reported for phases $\mathrm{I}^{26,27}$ and III, ${ }^{26}$ a positive linear compressibility in all the crystallographic directions is here observed for phase IV.

The coincidence of phases II and IV was further investigated by single crystal experiments. The P-T region indicated by Bridgman as pertaining to phase II was reached by two different P-T paths. In a first experiment we started heating phase I at $0.3 \mathrm{GPa}$ and we approached Bridgman phase II at $396 \mathrm{~K}$ by slowly increasing pressure and temperature to avoid phase III. The transition was observed above $0.7 \mathrm{GPa}$ by eye with the sudden powdering of the crystal. The second experiment was performed by compressing first phase I at room temperature. The single crystal overcame the transition to phase III unaltered in quality, then it was isobarically heated at 1.41.5 GPa. The first reflections relative to phase IV appeared at $378 \mathrm{~K}$ but the complete transformation of the pattern was achieved only at $408 \mathrm{~K}$. The sample was then cooled measuring diffraction pattern every $4-5 \mathrm{~K}$ and taking care of avoiding any pressure increase in order to explore the IV-III phase transition. The transformation to phase III occurred around $310 \mathrm{~K}$ at $1.3 \mathrm{GPa}$ as revealed by the patterns shown in Figure 3. This experiment therefore provides conclusive information about the $\mathrm{P}-\mathrm{T}$ range where phases III and IV are thermodynamically stable and of the coincidence between phase IV and Bridgman phase II, which hereafter we will indicate as IV.

\section{$3.2 \quad$ Spectroscopic data}

The transition from phase I to phase III, reported to occur at $293 \mathrm{~K}$ above $0.48 \mathrm{GPa},{ }^{2,8}$ is observed by IR absorption spectra at $0.45 \mathrm{GPa}$ where the 
coexistence of the two phases can be observed, as shown in Figure 4. From a spectral point of view, phase III is characterized, among other more subtle differences, by the appearance of a strong band in the N-H stretching region at lower frequency with respect to phase I. The same occurrence was also reported in the Raman spectrum, ${ }^{11}$ and we see this band is indicative of this phase. This band likely derives from the non chelate $\mathrm{N}-\mathrm{H} \cdots \mathrm{O}$ hydrogen bond, which is shorter in phase III than in both phases I and IV (ref. 2), thus causing a sudden red shift of the corresponding $\mathrm{N}-\mathrm{H}$ stretching band. Further compression of the sample determines appreciable changes of the spectrum occurring mainly between 2.0 and $8.0 \mathrm{GPa}$. This behavior can be appreciated in Figure 5. Some features, such as the two broad bands at 658 and $3171 \mathrm{~cm}^{-1}$, first intensify to then successively disappear at pressures exceeding 7-8 GPa, some new bands intensify to become characteristic of the high pressure spectrum (for example the one at $1150 \mathrm{~cm}^{-1}$ ), whereas yet other bands progressively weaken $\left(1453 \mathrm{~cm}^{-1}\right)$ until, in some cases (such as the doublet at $1090 \mathrm{~cm}^{-1}$ ), disappear completely.

Better information can be derived by the pressure evolution of the peak frequencies that have been obtained by fitting the absorption bands with pseudo-Voigt profiles. The most relevant information concerns the pressure evolution of the frequencies of the collapsed doublets relative to the symmetric CN stretching mode at about $1000 \mathrm{~cm}^{-1}$, and the $\mathrm{NH}_{2}$ asymmetric and symmetric bending modes at about 1080 and $1150 \mathrm{~cm}^{-1}$, respectively, all reported in Figure 6 (assignment from ref. 24). The frequency of the Davydov components of the symmetric CN stretching mode show a non linear evolution below $8 \mathrm{GPa}$ with a marked slope change around $3 \mathrm{GPa}$. At $8 \mathrm{GPa}$ 
both frequencies exhibit a clear discontinuity (about $5 \mathrm{~cm}^{-1}$ ). At nearly the same pressures we can observe an abrupt slope sign inversion for the the components of the $\mathrm{NH}_{2}$ symmetric bending mode. In particular, the high frequency component red shifts of about $25 \mathrm{~cm}^{-1}$ between 3 and $8 \mathrm{GPa}$ suggesting a dramatic change of the hydrogen bonds arrangement. A red shift of a Raman component of the $\mathrm{N}-\mathrm{H}$ stretching mode was also reported by Lamelas et al. in the same pressure range. ${ }^{11} \mathrm{~A}$ new component at $1060 \mathrm{~cm}^{-1}$ is instead observed above $3 \mathrm{GPa}$ in the $\mathrm{NH}_{2}$ asymmetric bending mode region. The pressures where these changes occur agree nicely with the III to IV $(2.8 \mathrm{GPa})$ and the IV to $\mathrm{V}(7.2 \mathrm{GPa})$ transitions reported in room temperature compression experiments monitored by both $\mathrm{X}$-ray ${ }^{2}$ and neutron diffraction. ${ }^{10,12,13}$ On the contrary, we do not have any indication of a transition occurring at $5 \mathrm{GPa}$ as indicated by Raman studies. ${ }^{11} \mathrm{~A}$ clear discontinuity of the frequency evolution of both components of the $\mathrm{NH}_{2}$ symmetric bending mode is also observed just above $15 \mathrm{GPa}$ possibly indicating another phase transition; however this is not supported by any other vibrational mode frequency evolution with pressure.

In order to verify the X-ray diffraction results obtained by heating the sample above $400 \mathrm{~K}$, we performed an isobaric heating experiment at $1.9 \mathrm{GPa}$ measuring IR absorption spectra every $5-10 \mathrm{~K}$. Some of these spectra are reported in the upper panels of Figure 7. Sudden spectral changes are observed above $370 \mathrm{~K}$ thus suggesting that they could be related to the transition to phase IV, since this temperature is almost the same where the transition was observed in the single crystal diffraction experiment. The coexistence of phases III and IV can be appreciated in the spectrum measured at $376.0 \mathrm{~K}$, 
whereas the spectrum measured $5 \mathrm{~K}$ higher in temperature shows the complete formation of phase IV. Besides the spectral changes occurring in the fingerprint region, it should be remarked the strong band at $3170 \mathrm{~cm}^{-1}$ disappears, anticipated to characterize phase III. Fitting with pseudo-Voigt profiles of selected spectral regions gives clear evidence-from the frequency evolution with temperature reported in Figure 8 - of the first order nature of the III-II transition. This result does not fully agree with the single crystal X-ray diffraction experiment where the transition completion was observed only $30 \mathrm{~K}$ higher in temperature. These FTIR absorption experiments were carried out on the sample powder loaded without any compression medium, such that non hydrostatic conditions could effectively contribute to alter transformation kinetics.

Once phase IV has been produced, the temperature was slowly lowered to ambient conditions (lower panels of Figure 7) but no spectral changes were observed during the cooling process thus confirming our diffraction experiment results. Phase IV is therefore the thermodynamic stable phase at ambient temperature also at pressures much lower than those reported in the literature. ${ }^{2,10}$ The III-IV transition appears therefore to be kinetically controlled and evidence of that is provided by the comparison between the spectra measured compressing at ambient temperature phase III and phase $\mathrm{IV}$, the latter after its attainment at $380 \mathrm{~K}$ and $1.9 \mathrm{GPa}$. The spectra measured at ambient temperature at $2.1 \mathrm{GPa}$ in these two different experiments are compared in the lower panel of Figure 9. The spectral differences before (phase III) and after the heating cycle (phase IV) are evident. Remarkably, once the quenched phase IV is compressed at ambient temperature, the 
spectrum does not show any of the changes previously described occurring at $3 \mathrm{GPa}$ and invoked to be characteristics of the formation of phase IV. For example, the new bands appearing in the spectrum $\left(1060\right.$ and $\left.1150 \mathrm{~cm}^{-1}\right)$ of phase III and taken as evidence of the III-IV phase transition (see upper panel of Figure 9), are already dominating the spectrum when the quenched phase IV is compressed. The coexistence of the two phases at a pressure where only phase IV should be present is therefore an indication of the large metastability range of phase III. It should be remarked that this behavior cannot be ascribed to non hydrostatic compression as the same pressure range was also reached in XRD experiments with the powder compressed with Ne.

Finally, the fit of the crystal components of the $\mathrm{NH}_{2}$ symmetric bending mode gives very reproducible results, as the frequencies are concerned, of those compressing phase III. This is a clear evidence that the crystal components under consideration are relative to phase IV and that the phase coexistence is not relevant as the frequency evolution is concerned. These results are compared in the lower panel of Figure 10 showing that the softening observed below $7 \mathrm{GPa}$ is perfectly reproducible being related to the structural changes occurring during the compression of phase IV. The transition to phase $\mathrm{V}$-besides from the abrupt slope inversion particularly evident for the high frequency component - is also revealed by the sudden intensity exchange between the two components as shown in the upper panel of Figure 10. 


\section{Discussion}

By combining IR absorption spectroscopy, powder and single crystal X-ray diffraction we have characterized the phase diagram of urea in a large pressure and temperature range. The most important novelty of this study is the employment of temperatures in excess of $400 \mathrm{~K}$ to study the close relation between hydrogen bonding and structural transformation kinetics. Hydrogen bonds govern many transformation processes, selecting specific paths for crystal structure changes, self assembly, and chemical reactions. Due to their relative weakness and flexibility they are extremely sensitive to the application of external pressure. In H-bonded systems significant metastability of the crystal structure is reasonably expected because of the necessity to overcome kinetic barriers related to the H-bond (re)arrangement. In this context nice examples are represented by pyridine and nitromethane. In pyridine the compression of the fluid at room temperature does not bring to the formation of the thermodynamically stable phase I, but the supercompressed fluid can crystallize either in the higher pressure phase II or give rise to a glass. ${ }^{6}$ In nitromethane, a continuous molecular rearrangement, which does not modify the crystal structure, extends along several GPa. ${ }^{5}$ Urea appears from this point of view as an important benchmark to identify and accurately characterize the P-T domains of the different crystal phases. As already mentioned, temperature is the key tool to overcome kinetic barriers and a precise information on the kinetics role can be gained by comparing room temperature compression experiments in annealed and not annealed samples.

The main results of this study are summarized in the phase diagram reported in Figure 11. First of all, Bridgman phase II and room temperature 
phase IV coincide. As already stated, here we refer to it as phase IV only. The formation of phase IV is kinetically controlled, and in fact the coexistence of phases III and IV extends to a large pressure range (about $2 \mathrm{GPa}$ ), as determined by X-ray diffraction on powder hydrostatically compressed in $\mathrm{Ne}$ in room temperature compression experiments. Once phase IV is produced by heating the sample at pressures exceeding $1 \mathrm{GPa}$, the crystal transforms back to phase III only around $1.3 \mathrm{GPa}$, close to ambient temperature, thus reducing considerably the thermodynamic stability range of phase III.

As discussed by Olejniczak et al., ${ }^{2}$ the structures of phases I, III, and IV are closely related with the molecules hydrogen-bonded into a 3D network with chelate bonds arranged along the [001] direction. In phase I this arrangement gives rise to channel voids along the [001] direction which collapse at the I-III phase transition because of the molecules tilt with respect to this direction. Asymmetry in the H-bonds network is introduced by the tilt which makes the two $\mathrm{N}-\mathrm{H} \cdots \mathrm{O}$ chelate bonds inequivalent, and results in the breakage of one of the two-center H-bonds reducing to three the number of $\mathrm{O} \cdots \mathrm{H}$ bonds. The fourfold coordination of the $\mathrm{O}$ atom is recovered in phase IV but with a strong reduction of the $a$ axis which makes this phase a collapsed version of phase I. Remarkably, in phase IV at $3.1 \mathrm{GPa}$ the $\mathrm{H}$...O distances are almost the same than in phase I $(0.5 \mathrm{GPa})$ and approximately $5 \%$ longer than in phase III $(1.5 \mathrm{GPa}) .{ }^{2}$ The molecular tilt proper to phase III determines a reduction in the structural compactness but, on the other hand, there is a strengthening of the three H-bonds across the I-III transition. These changes in H-bonding may be probed most accurately with spectroscopic techniques as exact hydrogen positions cannot be refined from 
X-ray crystallographic data. So, this strengthening is likely the cause of the abrupt red shift of the $\mathrm{N}-\mathrm{H}$ stretching component shown in Figures 4 and 7. The large metastability P-T region of phase III, which extends, according to the present study at least up to $420 \mathrm{~K}$ at $4 \mathrm{GPa}$, is likely due to the energy necessary to destabilize these H-bonds and recover the more symmetric local arrangement. The remarkable temperature increase necessary to overcome this barrier could suggest the participation of vibrational lattice modes or low-frequency internal modes to the reconstruction of the H-bond network of phase IV.

The lowest frequency internal modes are out-of-plane deformations of the $\mathrm{NH}_{2}$ groups around $500 \mathrm{~cm}^{-1}$ (ref. 28). The energy of these modes is rather high to allow a substantial modification of the population by a temperature around $400 \mathrm{~K}$. More reasonable is instead the active participation of high frequency external modes which around $3 \mathrm{GPa}$ extend up to $250 \mathrm{~cm}^{-1}$ (ref. 11), and have been assigned in phase I to librational modes. ${ }^{29} \mathrm{~A}$ large coexistence is revealed by X-ray diffraction also for the IV and V phases, for which a sluggish transition starting around $6 \mathrm{GPa}$ and extending for about $3 \mathrm{GPa}$ is measured by the simultaneous observation of the diffraction patterns of phases IV and V. The structural information obtained on phase $\mathrm{V}$ is not of the quality required to solve atomic positions and it is therefore not possible to quantitatively discuss the transition in terms of relative molecular orientation, but it is obvious that such metastability could be again ascribed to the H-bonding arrangement.

Lamelas and coworkers (ref. 11) reported the presence of crystal domains exhibiting different Raman spectra both in phases III and IV. They ex- 
plained this occurrence on the basis of different orientations of the same crystal structure. Coexistence of different phases was ruled out because of the large pressure ranges (several GPa) where the Raman bands assigned to different crystal orientations were observed. This was judged rather unlikely even more for the fact that such situation occurs in two different phases (IV and V). On the contrary, our results clearly show that large metastability ranges occur for all the three phases studied here. In addition, the very reproducible results obtained with both hydrostatic compression media (neon in this study; methanol/ethanol/water mixture in ref. 2; and deuterium in ref. 10), and no compression medium, clearly indicates that the metastability is not due to non hydrostatic conditions.

\section{Conclusions}

High-pressure high-temperature synchrotron ADXRD and FTIR absorption experiments on powders and single crystals of urea revealed the decisive role of H-bond in governing structural transformations. The structures observed are in fact strictly related to the H-bonding arrangement, especially of $\mathrm{N}-\mathrm{H}$... O that determines the relative molecular orientation. As different phases are characterized by the different number, strength and orientation of H-bonds, large energetic barriers must be overcome to accomplish the transitions which appear therefore kinetically controlled. The large energetic barrier separating phases III and IV is highlighted by the huge metastability P-T region of phase III. The thermodynamic boundary of this phase at ambient temperature is close to $1.3 \mathrm{GPa}$, but phase III persists above $4 \mathrm{GPa}$ if the 
sample is not annealed above $400 \mathrm{~K}$. This occurrence may be the reason that the high temperature Bridgman phase II was considered as a different phase from the ones existing at room temperature. Here we verify that the Bridgman phase II in fact coincides with phase IV. A large metastability range is also observed at the IV-V transition, suggesting that a further rearrangement of the H-bonding network also occurs in this case. Further investigations directed to characterize the structure and boundaries of phase $\mathrm{V}$ would be of interest. The knowledge of this phase diagram - and more specifically of the structural properties - is a prerequisite in view of a characterization of the high-temperature pressure induced chemistry in crystalline urea.

Acknowledgements This work is supported by Deep Carbon Observatory initiative (Extreme Physics and Chemistry of Carbon: Forms, Transformations and Movements in Planetary Interiors from the Alfred P. Sloan Foundation) and by the Italian Ministero dell'Istruzione, dell'Università e della Ricerca MIUR (grants FIRB - Futuro in Ricerca 2010 RBFR109ZHQ). The authors acknowledge the European Synchrotron Radiation Facility for provision of beamtime (proposal CH4708). K.D. gratefully acknowledges the Polish Ministry of Science and Higher Education for financial support through the "Mobilność Plus" program.

Author Information Corresponding Author: Roberto Bini, tel. +390554572489; E-mail: roberto.bini@unifi.it. 


\section{References}

[1] Dauber, P.; Hagler, A. T. Crystal Packing, Hydrogen Bonding, and the Effect of Crystal Forces on Molecular Conformation Acc. Chem. Res. 1980, 13, 105-112.

[2] Olejniczak, A.; Ostrowska, K.; Katrusiak, A. H-Bond Breaking in HighPressure Urea J. Phys. Chem. C 2009, 113, 15761-15767.

[3] Fanetti, S.; Citroni, M.; Bini, R. Pressure-Induced Fluorescence of Pyridine. J. Phys. Chem. B 2011, 115, 12051-12058.

[4] Citroni, M.; Datchi, F.; Bini, R.; Di Vaira, M.; Pruzan, P.; Canny, B.; Schettino, V. Crystal Structure of Nitromethane up to the Reaction Threshold Pressure J. Phys. Chem. B 2008, 112, 1095-1103.

[5] Citroni, M.; Bini, R.; Pagliai, M.; Cardini, G.; Schettino, V. Nitromethane Decomposition under High Static Pressure. J. Phys. Chem. B 2010, 114, 9420-9428.

[6] Fanetti, S.; Citroni, M.; Bini, R. Structure and Reactivity of Pyridine Crystal Under Pressure. J. Chem. Phys. 2011, 134, 204504.

[7] Swaminathan, S.; Craven, B. M.; McMullan, R. K. The Crystal Structure and Molecular Thermal Motion of Urea at 12, 60 and $123 \mathrm{~K}$ from Neutron Diffraction Acta Cryst. 1984, B40, 300-306.

[8] Bridgman, P. W. Polymorphism at High Pressures Proc. Am. Acad. Arts Sci. 1916, 52, 91-187. 
[9] Bonin, M.; Marshall, W. G.; Weber, H. P.; Toledano, F. Polymorphism in Urea. Annual Report 1998; ISIS Pulsed Neutron and Muon Source, Rutherford Appleton Laboratory: Didcot, U.K., 1998; 34-35.

[10] Donnelly, M.; Bull, C. L.; Husband, R. J.; Frantzana, A. D.; Klotz, S.; Loveday, J. S. Urea and Deuterium Mixtures at High Pressures $J$. Chem. Phys. 2015, 142, 124503.

[11] Lamelas, F. J.; Dreger, Z. A.; Gupta, Y. M. Raman and X-Ray Scattering Studies of High-Pressure Phases of Urea J. Phys. Chem. B 2005, 109, 8206-8215.

[12] Weber, H. P.; Marshall, W. G.; Dmitriev, V. High-Pressure Polymorphism in Deuterated Urea Acta Cryst. A 2002, 58, C174.

[13] Marshall, W. G.; Francis, D. J. Attainment of Near-Hydrostatic Compression Conditions Using the Paris-Edinburgh Cell J. Appl. Cryst. 2002, 35, 122-125.

[14] Miao, M. S.; van Doren, V. E.; Keuleers, R.; Desseyn, H. O.; van Alsenoy, C.; Martins, J. L. Density Functional Calculations of the Structure of Crystalline Urea Under High Pressure Chem. Phys. Lett. 2000, 316, 297-302.

[15] Góra, D.; Parliński, K. Phase Transitions of Deuterated $\mathrm{CO}\left(\mathrm{NH}_{2}\right)_{2}$ Under Pressure J. Chem. Phys. 2000, 113, 8138-8141.

[16] Méreau, R.; Desmedt, A. Mechanistic Aspects of the Solid-State Transformation of Ammonium Cyanate to Urea at High Pressure J. Phys. Chem. B 2007, 111, 3960-3967. 
[17] Mao, H. K.; Xu, J.; Bell, P. M. Calibration of the Ruby Pressure Gauge to 800 kbar Under Quasi-Hydrostatic Conditions J. Geophys. Res. 1986, 91, 4673-4676.

[18] Bini, R.; Ballerini, R.; Pratesi, G.; Jodl, H. J. Experimental Setup for Fourier Transform Infrared Spectroscopy Studies in Condensed Matter at High Pressure and Low Temperatures Rev. Sci. Instrum. 1997, 68, 3154-3160.

[19] Gorelli, F. A.; Ulivi, L.; Santoro, M.; Bini, R. The $\epsilon$ Phase of Solid Oxygen: Evidence of an $\mathrm{O}_{4}$ Molecule Lattice Phys. Rev. Lett. 1999, 83, 4093-4096.

[20] Prescher, C.; Prakapenka, V. DIOPTAS: a Program for Reduction of Two-Dimensional X-Ray Diffraction Data and Exploration. High Press. Res. 2015, 35, 223-230.

[21] Wojdyr, M. Fityk: a General-Purpose Peak Fitting Program J. Appl. Crystallogr. 2010, 43, 1126-1128.

[22] Petřiček, V.; Dušek, M.; Palatinus, L. Crystallographic Computing System JANA2006: General features. Z. Kristallogr. 2014, 229, 345-352.

[23] Klotz, S.; Chervin, J. C.; Munsch, P.; Marchand, G. L. Hydrostatic Limits of 11 Pressure Transmitting Media J. Phys. D: Appl. Phys. 2009, 42, 075413.

[24] Keuleers, R.; Desseyn, H. O.; Rousseau, B.; Van Alsenoy, C. Vibrational Analysis of Urea J. Phys. Chem. A 1999, 103, 4621-4630. 
[25] Vinet, P. Ferrante, J.; Smith, J. R.; Rose, J. H. Temperature Effects on the Universal Equation of State of Solids Phys. Rev. B 1987, 35, 1945-1953.

[26] Ostrowska, K.; Katrusiak, A. Giant Strain in Urea CMD 25 Paris 2014; https://cmd25jmc14.scienceconf.org/37788

[27] Miller, W.; Evans, K. E.; Marmier, A. Negative Linear Compressibility in Common Materials Appl. Phys. Lett. 2015, 106, 231903 .

[28] Rousseau, B.; Van Alsenoy, C.; Keuleers, R.; Desseyn, H. O. Solids Modeled by Ab-Initio Crystal Field Methods. Part 17. Study of the Structure and Vibrational Spectrum of Urea in the Gas Phase and in Its P421m Crystal Phase J. Phys. Chem. A 1998, 102, 6540-6548.

[29] Civalleri, B.; Zicovich-Wilson, C. M.; Valenzano, L.; Ugliengo, P. B3LYP Augmented with an Empirical Dispersion Term (B3LYP-D*) as Applied to Molecular Crystals CrystEngComm 2008, 10, 405-410. 


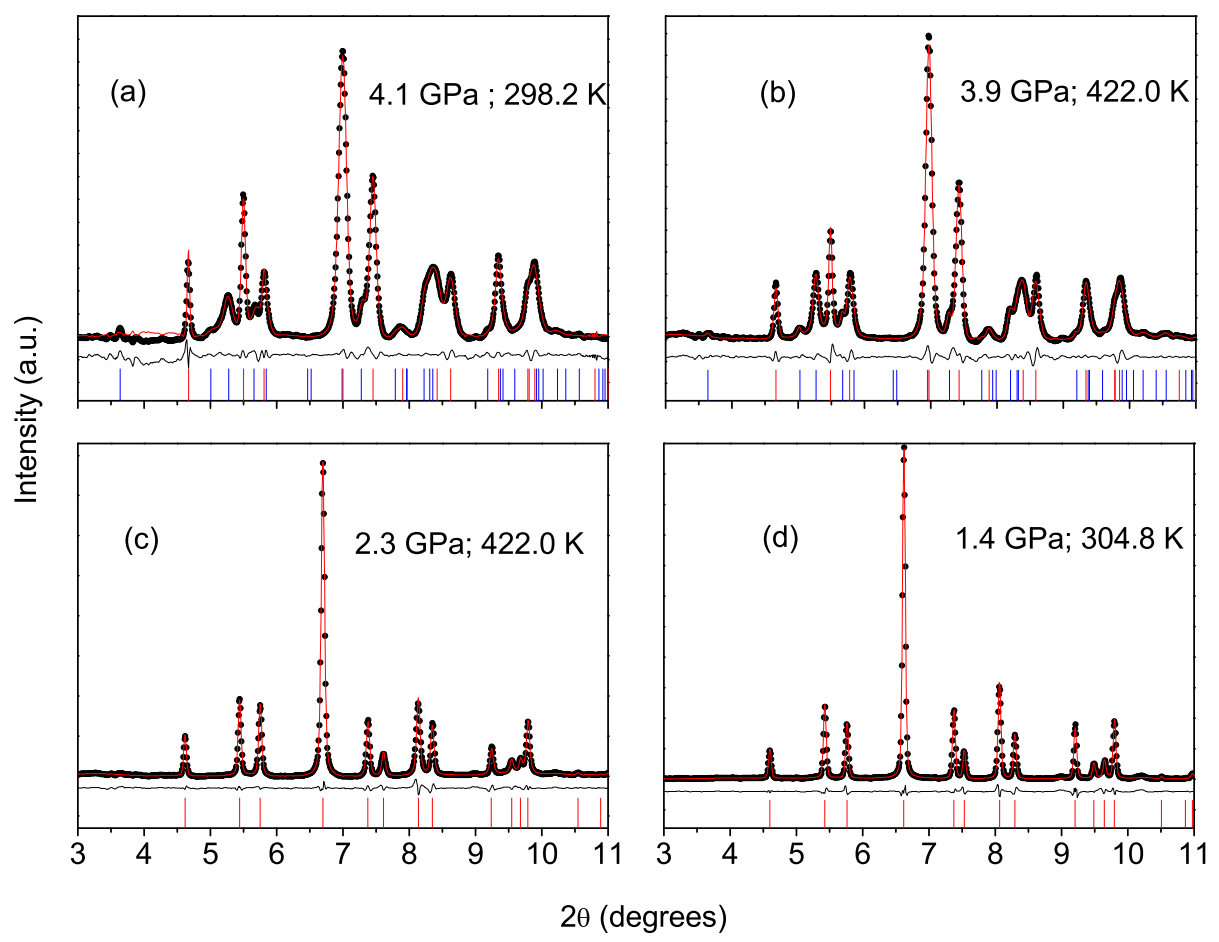

Figure 1: X-ray diffraction patterns $(\lambda=0.3738 \AA)$ of urea powder in Ne collected during (a) room temperature compression, followed by (b) an isobaric heating, (c) an isothermal decompression, and (d) a quasi-isobaric cooling cycle. Black dots are experimental data, red line is the Le Bail fit, and the black line is the difference between the two. The Le Bail fit has been performed following the assignment shown as tick marks: blue for the reflections of phase III $\left(P 2_{1} 2_{1} 2_{1}\right)$ and red for phase IV $\left(P 2_{1} 2_{1} 2\right)$. 

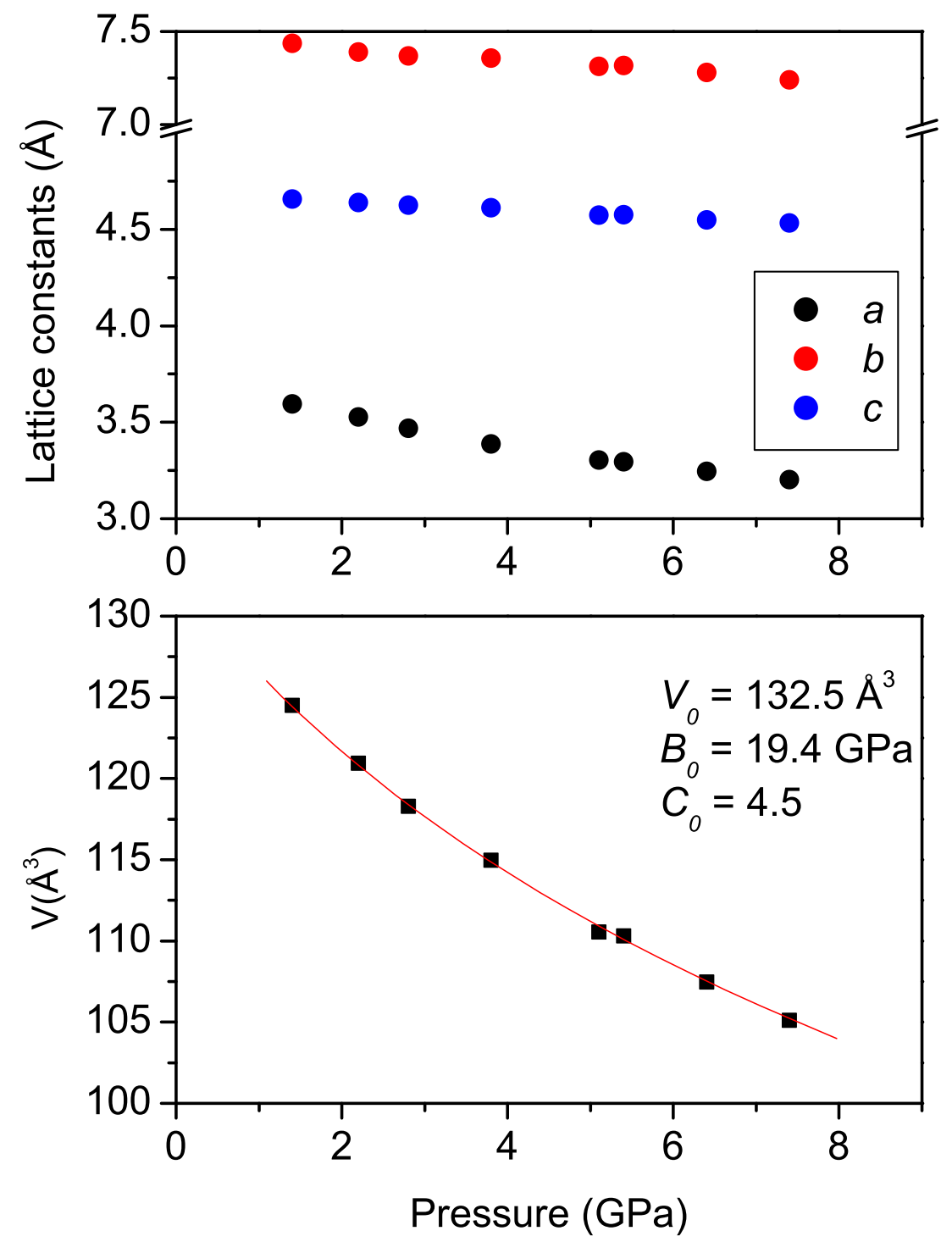

Figure 2: Pressure evolution of the lattice parameters and the volume of orthorhombic $P 2_{1} 2_{1} 2$ phase IV. The volume data have been fitted by a Vinet equation of state (eq. 1) with the fitting parameters reported in the figure. 


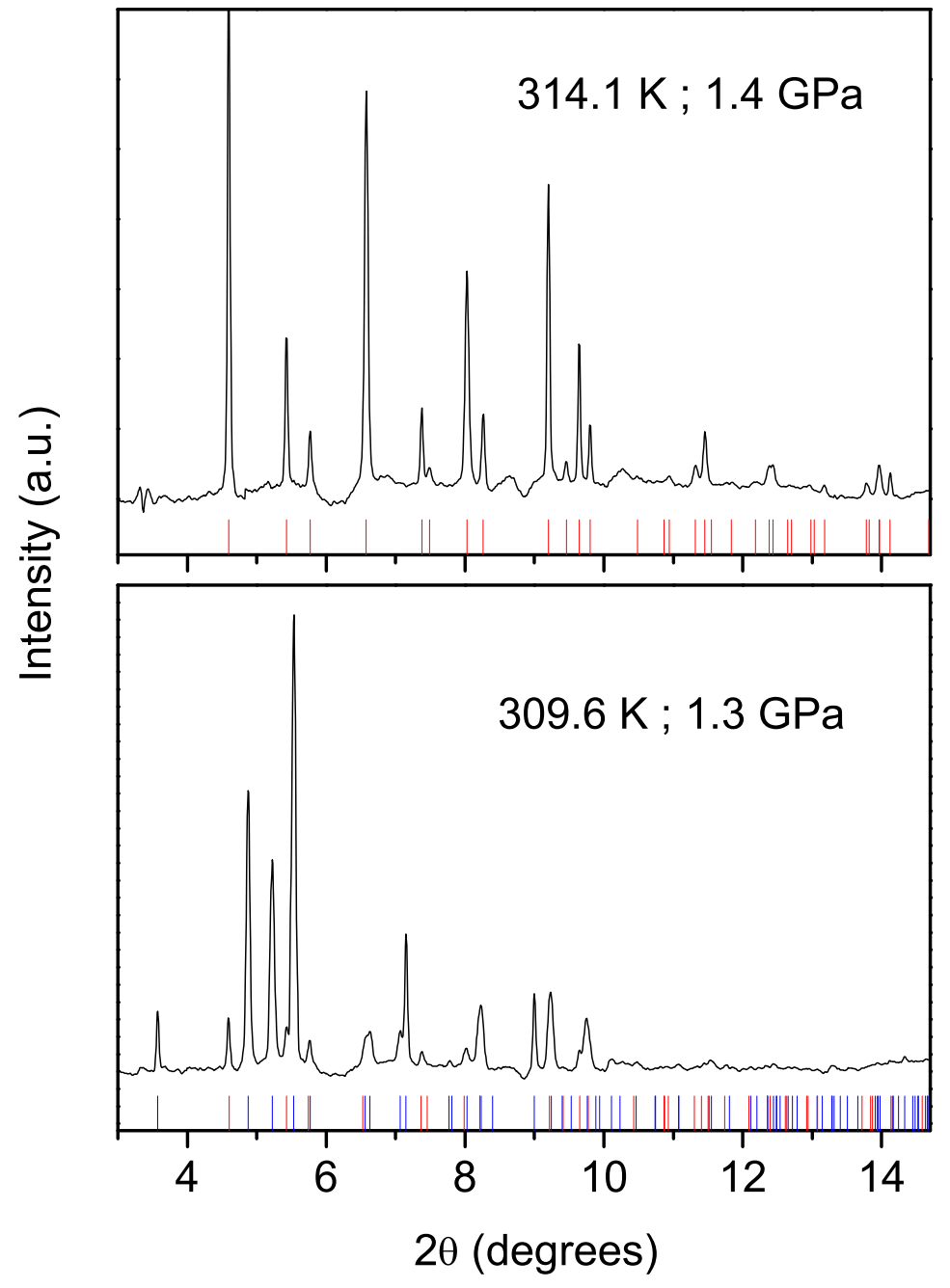

Figure 3: Integrated single crystal panoramic X-ray diffraction patterns $(\lambda=$ $0.3738 \AA$ ) measured on cooling phase IV (Bridgman phase II) through the IV-III transition. The reflections expected for the two phases are shown as blue (phase III) and red (phase IV) tick marks. 

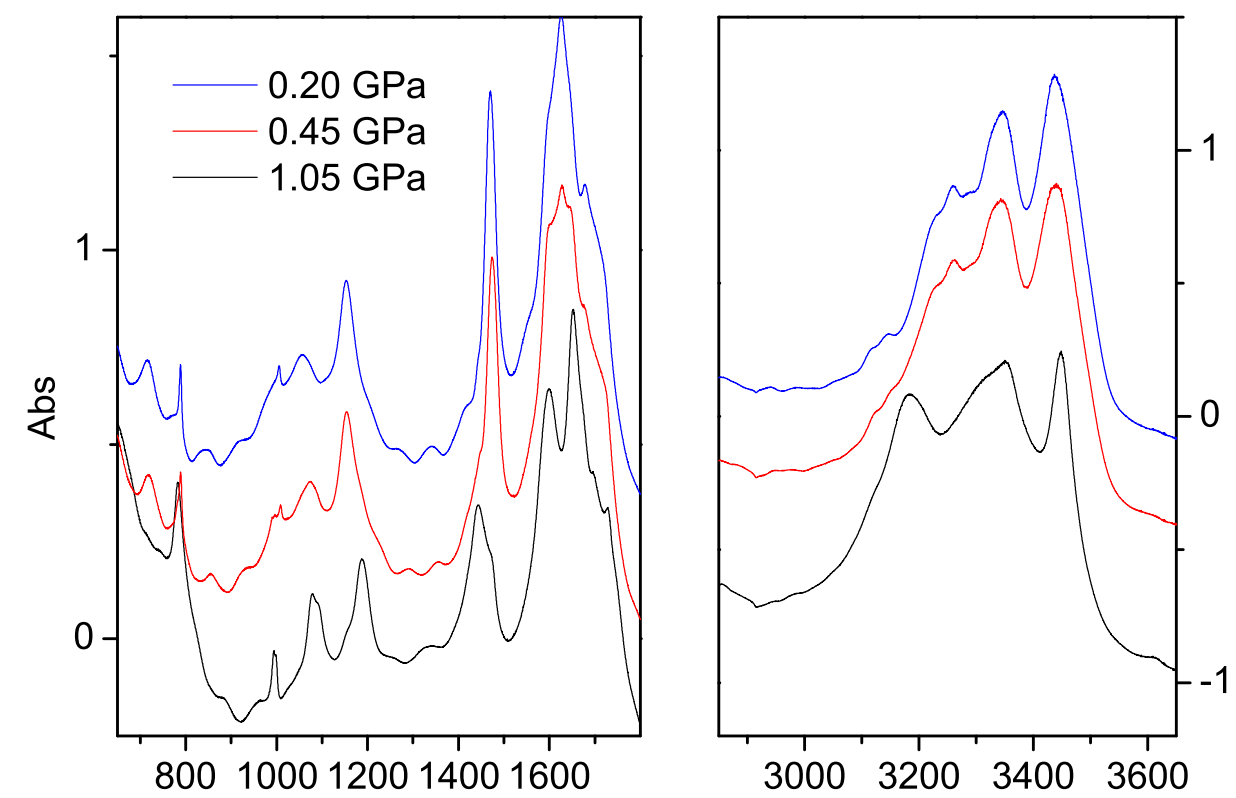

Frequency $\left(\mathrm{cm}^{-1}\right)$

Figure 4: FTIR absorption spectra through the I-III phase transition in the low (left panel) and high (right panel) frequency region. The spectra at 0.45 and 1.05 GPa exhibit spectral features of both crystal phases indicating their coexistence. 


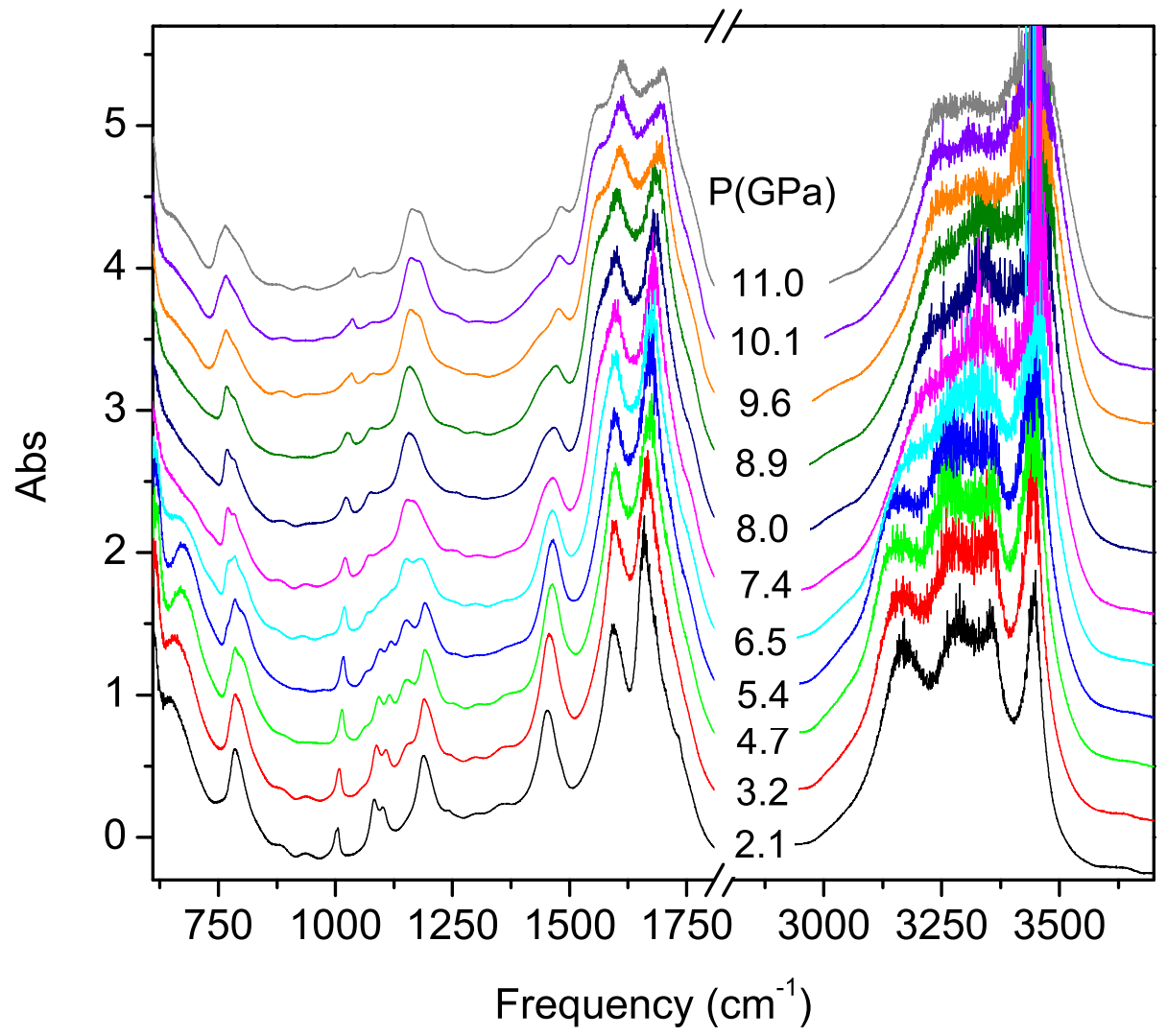

Figure 5: Pressure evolution of the FTIR absorption spectrum during room temperature compression experiment. 


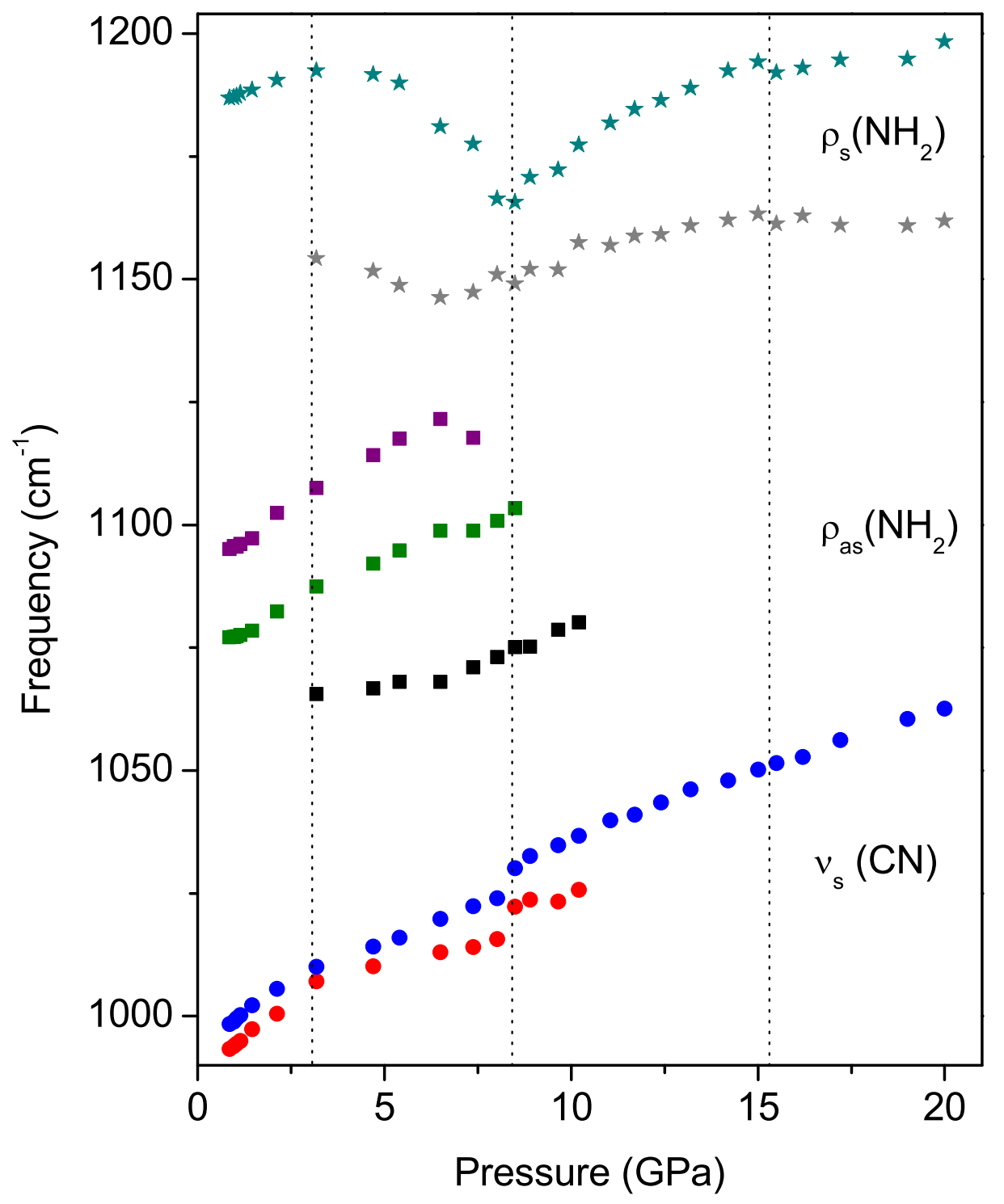

Figure 6: Pressure shift of the Davydov components relative to the CN stretching (dots), the $\mathrm{NH}_{2}$ asymmetric bending (squares) and $\mathrm{NH}_{2}$ symmetric bending (stars) modes. 

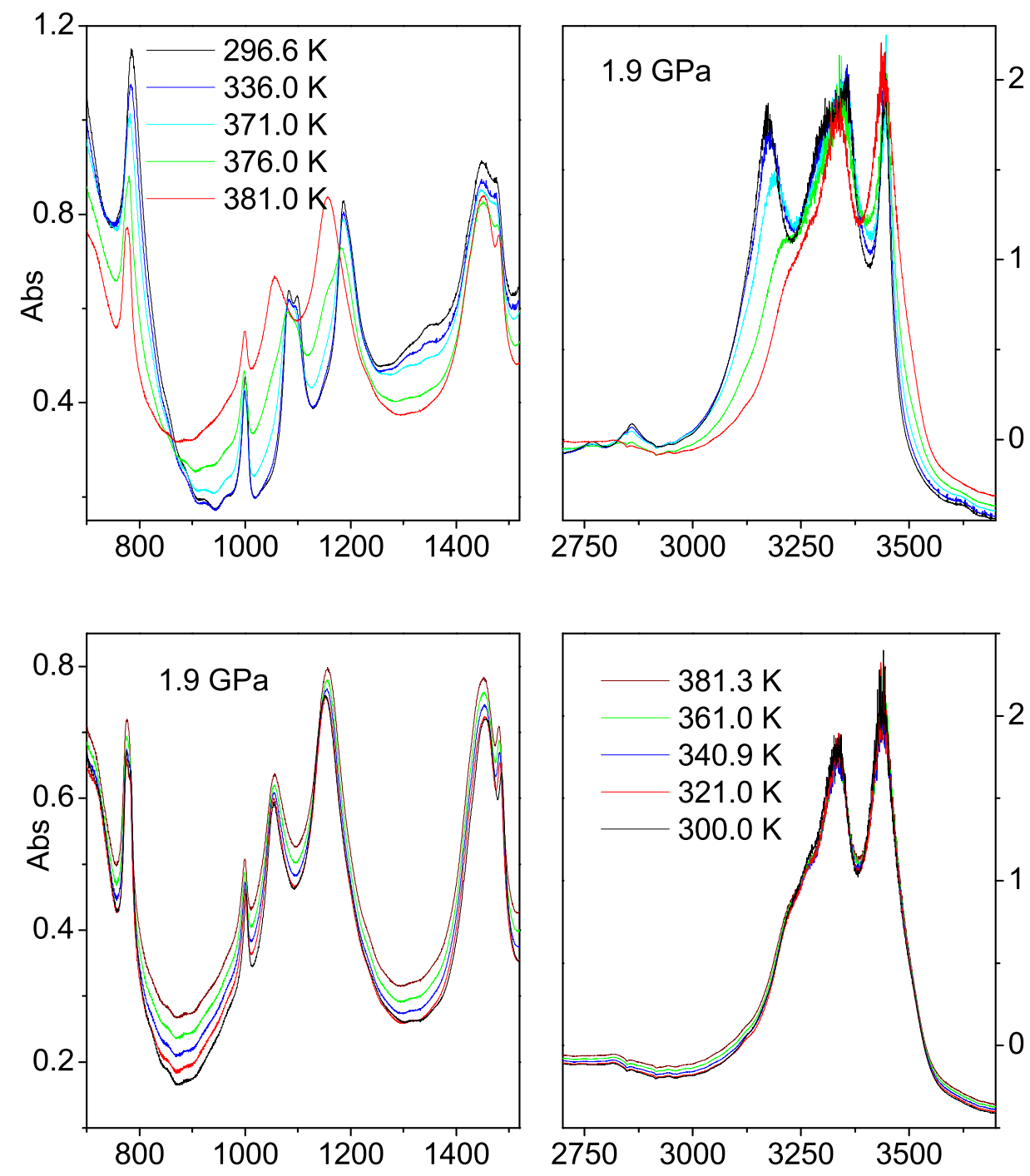

Frequency $\left(\mathrm{cm}^{-1}\right)$

Figure 7: Temperature evolution of selected IR absorption spectra regions during the isobaric heating (upper panels) and cooling (lower panels) cycles performed at $1.9 \mathrm{GPa}$. 


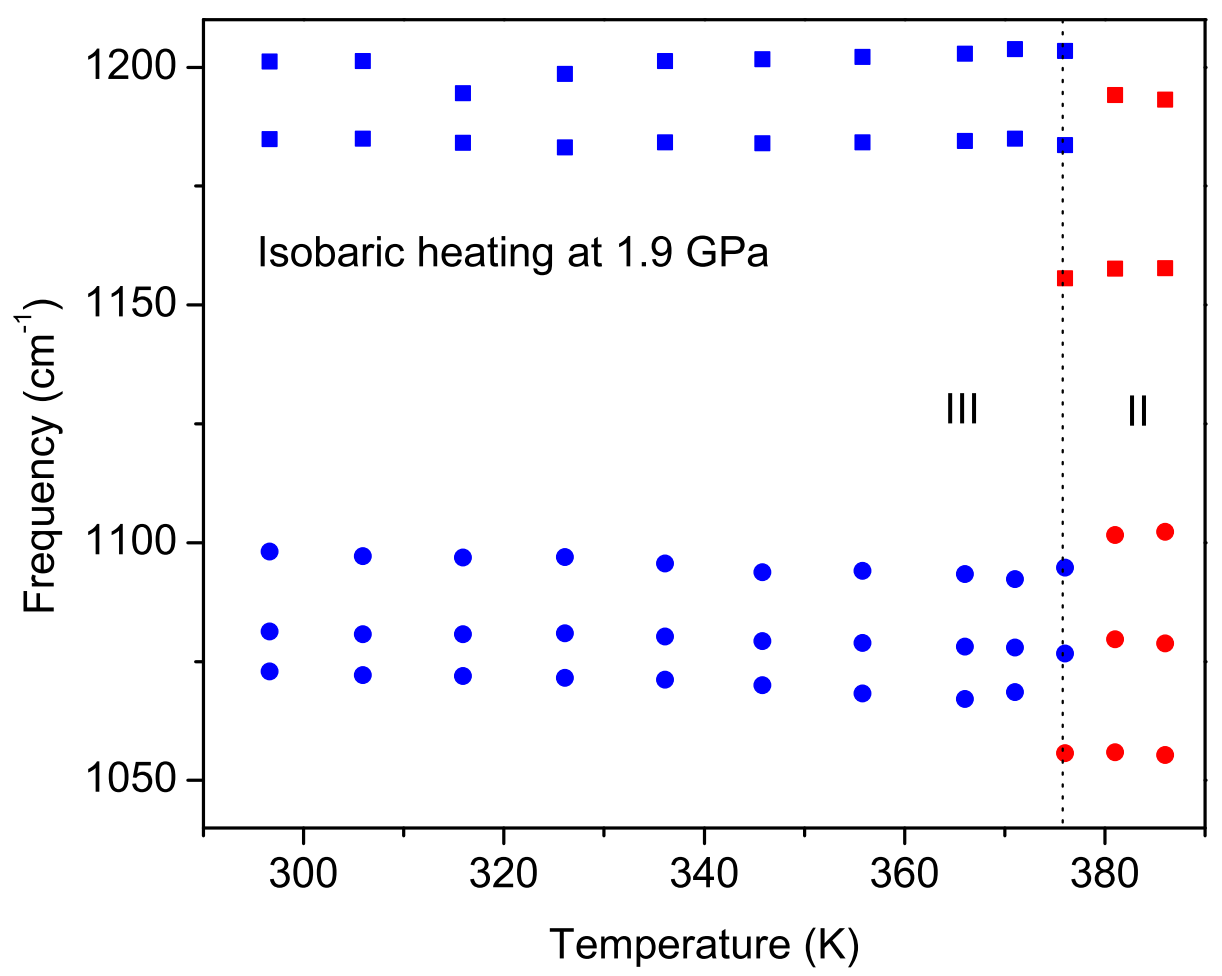

Figure 8: Temperature evolution of the peak frequencies relative to the Davydov components of the $\mathrm{NH}_{2}$ asymmetric bending (full dots) and $\mathrm{NH}_{2}$ symmetric bending (full squares) modes along the isobaric $(1.9 \mathrm{GPa})$ heating experiment. 


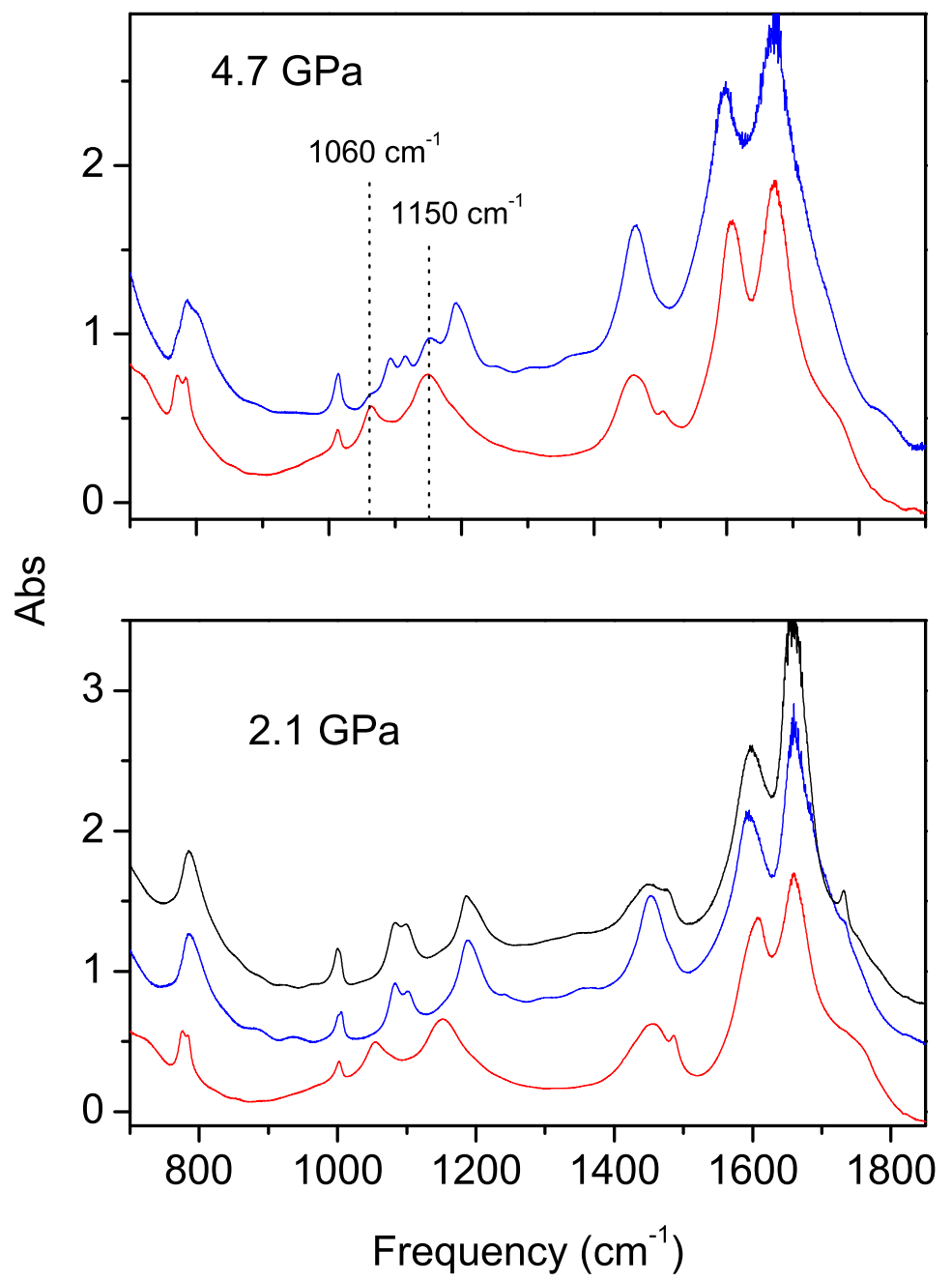


Figure 9: Lower panel: comparison of the spectra measured at ambient temperature and $2.1 \mathrm{GPa}$ in two different experiments. The blue trace has been measured during an ambient temperature compression experiment, the black and red traces have been measured in another experiment before and after, respectively, the isobaric heating cycle at $1.9 \mathrm{GPa}$ up to the attainment of phase IV. Upper panel: comparison of the spectra measured at ambient temperature and $4.7 \mathrm{GPa}$ in two different experiments. The blue trace has been measured during an ambient temperature compression experiment, the red trace has been measured in another experiment after performing an isobaric heating cycle at $1.9 \mathrm{GPa}$ up to the attainment of phase IV. Dotted lines indicate the peaks at 1060 and $1150 \mathrm{~cm}^{-1}$ discussed in the text. 

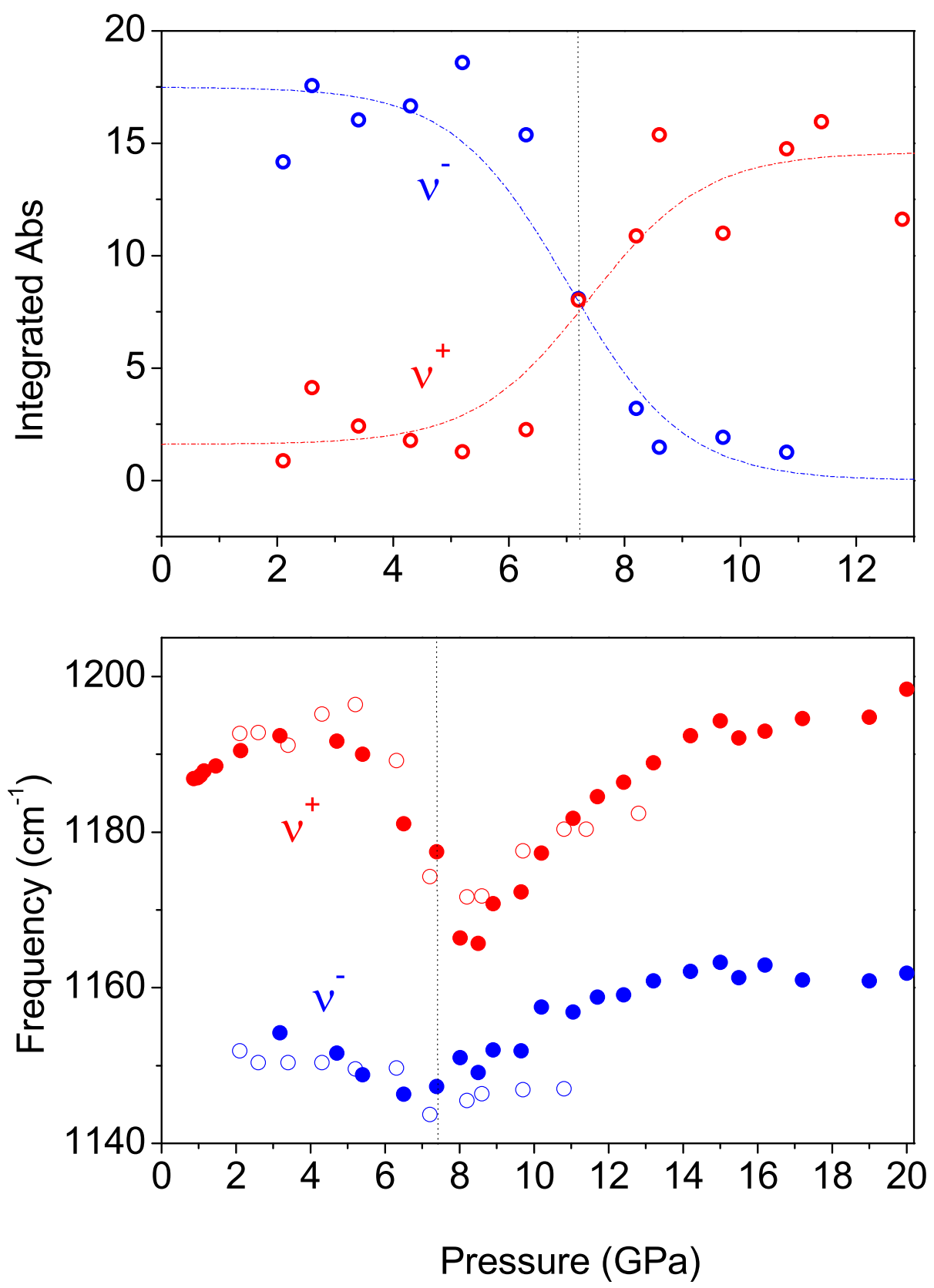
Figure 10: Lower panel: pressure shift of the $\mathrm{NH}_{2}$ symmetric bending Davydov components measured in two different ambient temperature experiments: compressing phase III without thermal annealing(full dots), compressing phase IV obtained at $1.9 \mathrm{GPa}$ and $380 \mathrm{~K}$ (empty dots). Upper panel: pressure evolution of the integrated area of the two components while compressing at ambient temperature the phase IV obtained at $1.9 \mathrm{GPa}$ and $380 \mathrm{~K}, \nu^{+}$and $\nu^{-}$indicate the high and low frequency component, respectively. 


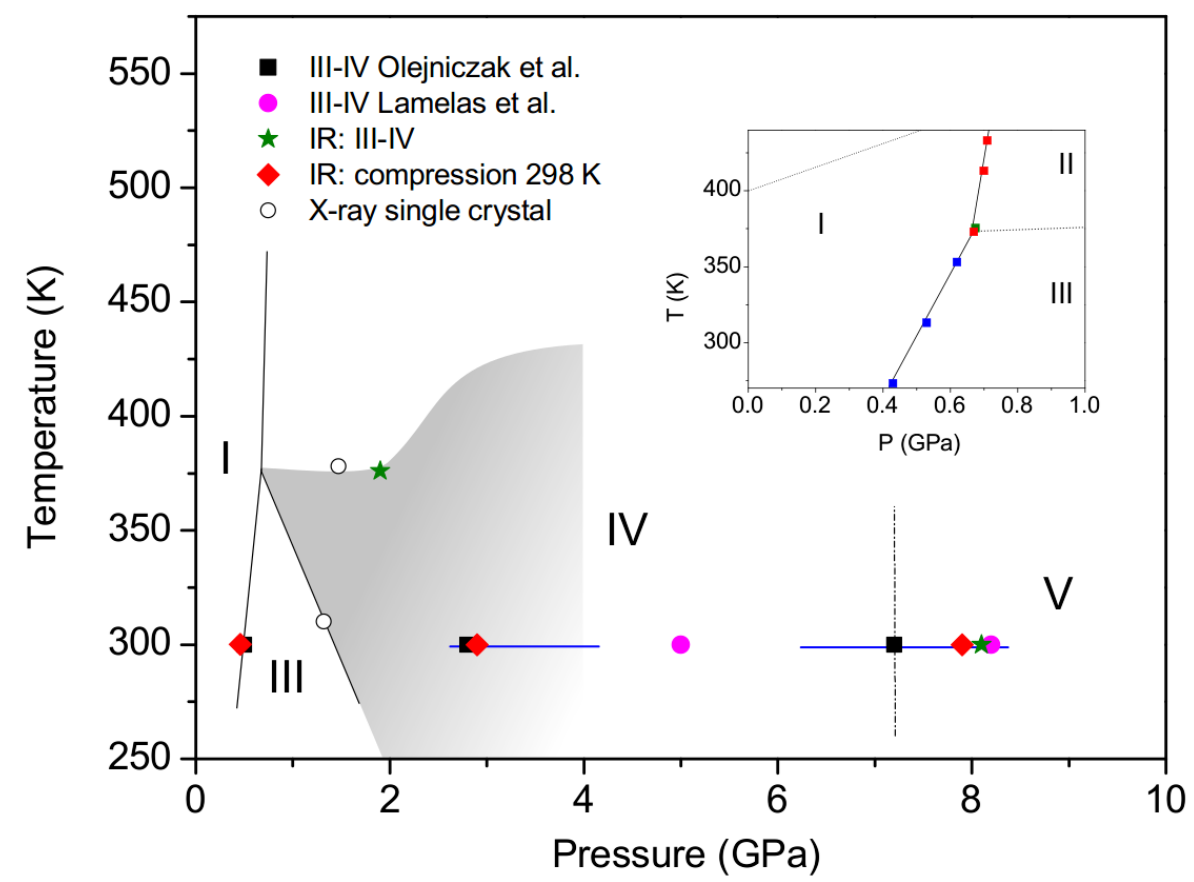

Figure 11: Phase diagram of urea after the present study and comparison with the portion studied by Bridgman, ${ }^{8}$ which is reported as an inset. The symbols indicate the transitions as reported in the literature and as deduced by this study. Blue full lines indicate the coexistence, observed in X-ray powder diffraction experiments compressed with Ne, of phases III and IV (room temperature compression) and of phases IV and V (after phase IV was completely transformed at high temperature). Bridgman phase II is not reported as it is shown to coincide with phase IV in this work. The shaded portion of the phase diagram indicates the metastability $\mathrm{P}-\mathrm{T}$ region of phase III detected in the present study. 